\title{
Development and Validation of a Questionnaire to Assess the Determinants of Dietary Adherence Among Patients After Bariatric Surgery
}

\author{
Hanfei Zhu',* \\ Ziqi $\operatorname{Ren}^{2, *}$ \\ Hongxia $\mathrm{Hua}^{3}$ \\ Kang Zhao (D) \\ Lingyu Ding' \\ Shuqin Zhu' \\ Ningli Yang ${ }^{3}$ \\ Hui Liang ${ }^{3}$ \\ Qin $X u^{1}$
}

'School of Nursing, Nanjing Medical University, Nanjing, Jiangsu, People's Republic of China; ${ }^{2}$ School of Nursing, Fudan University, Shanghai, People's Republic of China; ${ }^{3}$ Department of Bariatric and Metabolic Surgery, the First Affiliated Hospital of Nanjing Medical University, Nanjing, Jiangsu, People's Republic of China

*These authors contributed equally to this work
Correspondence: Qin Xu

School of Nursing, Nanjing Medical University, 818 Tianyuan East Road, Jiangning District, Nanjing, Jiangsu, 210029, People's Republic of China

Tel +86-25-86869553; +86-1360I587208

Fax $+86-25-86869555$

Email qinxu@njmu.edu.cn
Purpose: This study was aimed to develop a questionnaire to assess the determinants of dietary adherence among patients after bariatric surgery based on the attitude-social influence-efficacy (ASE) model and to evaluate its psychometric properties.

Patients and Methods: According to semi-structured interview, Delphi expert consultation and pilot study, the initial questionnaire was formed and applied to investigate 319 patients after bariatric surgery in a tertiary hospital in Jiangsu Province, China. The reliability and validity of the scale were tested.

Results: The 28-item of the attitude-social influence-efficacy questionnaire after bariatric surgery (ASEQBS) was formed. The results of exploratory factor analysis showed that four factors, including intention, attitude, social influence, and self-efficacy, could be extracted, and the cumulative variance contribution rate reached $59.98 \%$. Confirmatory factor analysis showed the model fit well. The content validity index of each item was $0.800-1.000$, and the content validity index of the ASEQBS was 0.857 . The total Cronbach's $\alpha$ of the ASEQBS was 0.920 , the split-half reliability was 0.774 , and the retest reliability was 0.922 .

Conclusion: The results suggest that ASEQBS is a valid and reliable measure of determinants of dietary adherence. It may be useful to evaluate the influence factors of dietary adherence and helpful to evaluate the efficacy of tailored dietary intervention programs.

Keywords: bariatric surgery, dietary adherence, attitude-social influence-efficacy model, questionnaire, validation

\section{Introduction}

Obesity is defined as abnormal or excessive fat accumulation and has been classified as a disease by the World Health Organization (WHO), which presents a risk to an individual's health. ${ }^{1}$ It is related to the development of cardiovascular diseases, endocrine diseases, and cancers., ${ }^{2,3}$ With the increasing prevalence around the world, obesity has become a globally public health problem. ${ }^{4}$ According to the WHO, it was estimated that the prevalence of obesity had been over $13 \%$ in 2016 and it will be increased by $33 \%$ by $2030 .{ }^{1}$ The Report on the Nutrition and Chronic Disease Status of Chinese Residents indicated that more than $50 \%$ of adult residents were overweight or obese in China. ${ }^{5}$ Due to the large population base, there are the most obese people in China and the total number of severe obese people is second only to the United States. ${ }^{6}$ Therefore, effective treatments are needed to reduce the negative impact of obesity on patients' health and the economic burden on society. 
Bariatric surgery (BS) has been considered a sustainable and effective treatment for severe obesity or related comorbidities, specifically Type 2 diabetes mellitus (T2DM). ${ }^{7}$ The most commonly used surgical procedures are sleeve gastrectomy (SG) and Roux-en-Y gastric bypass (RYGB). ${ }^{8}$ The alterations of gastrointestinal anatomy lead to food restriction and nutrient malabsorption to some extent, which may cause nutrition problems among postoperative patients. ${ }^{9}$ Anemia, dumping syndrome, osteoporosis, or even neurological problems are common in post-bariatric patients. ${ }^{10}$ In order to lose more weight and avoid postoperative complications, patients are advised sufficient protein, micronutrient supplementation, less carbohydrate and fat intake. ${ }^{11,12}$ However, patients' adherence to these dietary recommendations was not good and needed to be improved. ${ }^{13}$ Previous studies have revealed that postoperative patients generally suffered from insufficient water intake, failure to supplement vitamins and trace elements as required, and excessive intake of carbohydrates and fats. ${ }^{14}$ It was also demonstrated that patients with better adherence had a greater decrease in BMI, which meant adherence to recommended rules predicted the positive outcomes of bariatric surgery. ${ }^{15}$ Notwithstanding the fact that bariatric surgery has been increasingly popular in China, ${ }^{16}$ representative data on people's reasons for following dietary recommendations is still limited. Consequently, a greater understanding of determinants is important to take effective measures to improve dietary adherence.

Dietary adherence is a reflection of various factors, including socio-demographic factors, disease-related factors and psychosocial factors, among which psychosocial factors are the most important intervenable factors. ${ }^{17}$ Health behavior change theory is useful in explaining behavior through psychosocial factors, and interventions targeting these factors are often more effective. ${ }^{18}$ Specifically, Attitude-Social influence-Efficacy (ASE) model can help to systematically identify intrapersonal factors and interpersonal factors that can support behavioral modifications, making it easier to achieve the desired improvements. ASE model had been proposed by Dr.Hein de Vries in 1988, which integrated elements of the social learning theory and theory of planned behavior. ${ }^{19}$ It argues that people's intention to perform a behavior is the main predictor of actual behavior performance and intention depends on the individual's attitude, social influence, and self-efficacy. ${ }^{20,21}$ Intention to perform a behavior refers to the person's readiness to perform a behavior.
Attitude consists of the advantages and disadvantages of a particular behavior and could be assessed by measuring the beliefs that are associated with a particular behavior and the evaluations of these beliefs. ${ }^{22}$ Social influence could be defined as the subjective norm, injunctive norm, and descriptive norm. The subjective norm means that people have with respect to the specific behavior. Injunctive norm refers to a person's perception of support from others to perform or refrain from the behavior. Descriptive norm refers to a person's perception of whether the important people actually performs or refrains a certain behavior themselves. ${ }^{23}$ Self-efficacy can be seen as a person's belief whether he or she could perform the behavior and cope with barriers. Many studies have used the ASE model as a theoretical framework to analyze the determinants of eating behavior or adherence. Brug et $\mathrm{al}^{23}$ applied this model to explore the psychosocial determinants affecting Dutch people's fruit and vegetable consumption, and the results proved that low self-efficacy and less positive attitudes may lead to lower consumption of food. Pajor et $\mathrm{al}^{24}$ also demonstrated socio-cognitive factors in the ASE model are associated with dietary supplement use. Based on the ASE model, Zhang et al ${ }^{25}$ found that the intention of rational eating behavior was affected by attitude, social influence, and self-efficacy, and these were important factors that can be interfered with. Bolman et $\mathrm{al}^{26}$ also used the ASE model in exploring the influencing factors of medication adherence in asthma patients, and it could explain medication adherence well. All above studies suggested the feasibility and advantages of using the ASE model to analyze the determinants of dietary adherence. By using the ASE model, it could not only provide a theoretical framework for researchers to explain the mechanism of dietary adherence, but also provide a theoretical basis for clinical staff to design a tailored nutrition education plan for patients.

In the ASE model, since intention, attitude, social influence, and self-efficacy are latent variables, appropriate and effective instruments are needed to measure these factors. After measuring these factors accurately, it could be known about the extent of intention, attitude, social influence, and self-efficacy. Most studies have adopted general measurement tools, such as the Multidimensional Scale of Perceived Social Support ${ }^{17}$ and the General Perceived Self-efficacy Scale, ${ }^{27}$ but the concepts measured by these tools are inconsistent with the ASE model. In addition, the questionnaire applicable to the assessment of eating self-efficacy in patients undergoing bariatric 
surgery, such as the Weight Efficacy Lifestyle Questionnaire-Short Form, ${ }^{28}$ has not been verified in the Chinese post-bariatric patients, and this questionnaire mainly targets overeating, which is not completely consistent with the concept of dietary adherence defined by this study. What's more, only a few studies provided information about reliability and construct validity. Since these questionnaires are either combinations of different structures of other questionnaires or failure to use all of the ASE model constructs, or content irrelevance, there is a need for developing a culturally relevant questionnaire targeted post-bariatric population.

As a consequence, there still lacks a standardized measure of determinants of dietary adherence for Chinese patients after bariatric surgery based on the ASE model. To address this gap in knowledge, the purpose of this study is to develop a reliable and valid instrument to identify the determinants of dietary adherence and facilitate the improvement of adherence.

\section{Methods}

Two phases were conducted to develop a reliable and valid measurement tool, which was called Attitude-Social influence-Efficacy Questionnaire after Bariatric Surgery (ASEQBS). Phase 1 included the development of the initial version of the questionnaire by semi-structured interviews and the process of expert consultation using the Delphi method. Phase 2 entailed a formal crosssectional survey to test the reliability and validity of the questionnaire.

\section{Phase I: Development of the Initial Version of ASEQBS}

\section{Item Pool Formation}

Firstly, we conducted semi-structured interviews with 20 participants who had undergone bariatric surgery at least three months, which included 10 males and 10 females aged from 22 to 58 years. The participants were selected purposefully by maximum diversity sampling (eg, age, occupation, marital status, surgical method) to ensure independence and representativeness until data saturation. All interviews were conducted face-to-face individually by the first author (HFZ). The interview guiding questions included the following: (1) How well do you follow the dietary recommendations? (2) What are the advantages or disadvantages of following these dietary recommendations? (3) Who will influence you to follow the dietary recommendations? How do you think these people's thoughts or behaviors influence you? (4) What factors or circumstances will make it easy or difficult to adhere to dietary recommendations? (5) In addition to the above, what other reasons do you think will influence you to follow the dietary recommendations? For the development of the questionnaire, dietary adherence was defined as the extent to which patients follow dietary recommendations by health care professionals or dietitians, such as nutrient intake, water intake, eating habits and so on. ${ }^{21}$ For data analysis, all interviews were tape-recorded after obtaining informed consent. The interviews were conducted and transcribed in Chinese, and the transcriptions were used for content analysis. Themes were extracted using a deductive approach, which has three main phases, preparation, organization, and reporting. ${ }^{29}$ In the preparation phase, the whole text was read to immerse in the data and fully understand the data. Next, the organizing phase includes coding, creating categories, and abstraction. ${ }^{29}$ Finally, in the reporting phase, interpretation and analysis of results generated items of the questionnaire. Data analysis was independently performed by two of the researchers (HFZ and KZ), and consensus on results was achieved through discussion sessions among them. The details of the interview results are presented in the previous study. ${ }^{30}$ After discussion and revision repeatedly in research group, a list of 28 candidate items formed the first draft of the questionnaire, and each item was measured using a fivepoint Likert scale ranging from 1 to 5 .

\section{Expert Consultation}

Two rounds of Delphi anonymous consultation were conducted to test the content of the initial questionnaire. In the two rounds of expert consultation, 14 and 12 experts were invited respectively, and 12 experts agreed to participate. Therefore, a total of 12 experts evaluated the initial scale, and the same experts participated in the two rounds. The active coefficients of the experts in the two rounds were $85.71 \%, 100 \%$, respectively. 12 experts in surgery, nutriology, nursing, and health education were included, which comprised 2 males and 10 females, aged 24-54 (41.58 \pm 7.59 ) years old, who had worked in their role for an average of $17.50 \pm 8.92$ years, and 8 of them had master's or higher degrees. The anonymous consultation questionnaires were sent to the experts by email, which included the introduction of the study and the evaluation part of the ASEQBS. The evaluation part contained the importance and linguistic expression of each item. The importance 
was rated on a Likert-type scale from 1 (very unimportant) to 5 (very important). Comments and suggestions for item improvement were provided by the experts. The expert authority coefficient was 0.93 , and the Kendall concordance coefficients were 0.28 and 0.41 for each round. According to the results of expert consultation, two items "When I have a strong appetite, I can adhere to the diet" and "My fellow patients can adhere to the diet" were removed because the average score of importance assignment $<4.00$ or coefficient of variation $>0.25$. Four items were modified to make the item content more appropriate. Three items were added according to the experts' suggestions, "When I am in a good mood, I can adhere to the diet", "I intend to adhere to the diet", "I would like to make the effort to adhere to the diet". Finally, a draft ASEQBS including 29 items was generated.

\section{Pilot Study}

The pilot study was conducted to ensure that the items were comprehensible and unambiguous, which involved 20 participants through face-to-face investigation. After completing the questionnaire, the clarity of the items, the format of the questionnaire, and the time needed to complete were evaluated. Participants considered all the items in the questionnaire were clearly expressed and easy to understand. Thus, no other major adjustments had to be made. The mean time for completing the questionnaire was about $5 \mathrm{~min}$.

\section{Phase 2: Psychometric Tests of the Draft ASEQBS}

\section{Participants and Study Setting}

The participants were recruited by convenience sampling at the Department of Bariatric and Metabolic Surgery in a tertiary hospital in Jiangsu province from May to September 2020. Inclusion criteria were: (1) met the surgical criteria of the Chinese Guidelines for the Surgical Treatment of Obesity and Type 2 Diabetes: body mass index $(\mathrm{BMI}) \geq 32.5 \mathrm{~kg} / \mathrm{m}^{2}$ or $27.5 \leq \mathrm{BMI}<32.5$ with comorbidities; (2) underwent BS at least 3 months; (3) age $\geq 18$ years old. Exclusion criteria were: patients who (1) experienced serious complications, such as gastrointestinal bleeding, anastomotic fistula, or intestinal obstruction; (2) suffered from other serious diseases affecting eating behavior, such as hyperthyroidism, hypothyroidism, malignant tumors, or cardiovascular events; (3) were pregnant or lactating women; (4) underwent revisional surgery. The sample size was calculated according to the requirement of factor analysis, which required at least 5-10 participants per item. ${ }^{31}$ Besides, the sample size of exploratory factor analysis (EFA) should be at least 100, and that of confirmatory factor analysis (CFA) should be at least 150 . Considering $10 \%$ of the invalid questionnaires, the total sample size needed for this study was at least 315 . Ethical approval was granted from the ethical committee of the First Affiliated Hospital of Nanjing Medical University (Ethical Approval Code: 2020-SR-247). All participants signed an informed consent form for participating in the study. This study was conducted in compliance with the Declaration of Helsinki.

\section{Instrument}

The participants' demographic information was collected via self-reported, including age, gender, educational level, occupational status, and marital status. Anthropometric data, such as height and weight were also reported by participants and then BMI was calculated. Disease-related information was obtained from the electronic medical record.

The first draft of the 29-item ASEQBS was applied to assess the determinants of post-bariatric patients' dietary adherence during the last month. This questionnaire was self-reported and each item was rated from 1 to 5 points. Total scores ranged from 29 to 145 , with higher scores in each dimension indicating better intention, attitude, social influence, or self-efficacy.

\section{Data Collection}

Questionnaires were distributed face-to-face. A text explaining the purpose of the survey and data privacy would be informed to the participants. After obtaining informed consent, data was collected by two uniformly trained researchers (HFZ and KZ). All questionnaires were completed by the participants themselves, or with the help of the investigator. If participants did not understand the questionnaire well or were unable to fill it in by themselves, the investigator read each item in turn without any suggestion, and then recorded the choices made by the participants. The investigator collected the questionnaires immediately after participants filled them out, and then checked the completeness. If the participant randomly made the choice (in wavy or linear form) or gave up answering the questionnaire halfway, the questionnaire was considered to be invalid.

\section{Item Selection}

Item analysis was used to analyze and screen the item, and the criteria were as follows: ${ }^{32}(1)$ items with no statistically 
significant difference in scores between high and low groups or with a critical ratio $<3$; (2) items with standard deviation $<0.75$; (3) item-total correlation or corrected item-total correlation coefficient $<0.4$; (4) overall Cronbach's $\alpha$ for the questionnaire increased after deleting an item; (5) factor loading value $<0.4$ or communalities value $<0.2$. If the item meets at least three of the above six criteria for deletion, it will be deleted. ${ }^{33}$

\section{Reliability and Validity Test}

Construct and content validity were evaluated for the questionnaire. Construct validity was evaluated by EFA and CFA. Prior to conducting EFA, the Kaiser-MeyerOlkin (KMO) measurement of sampling adequacy and Bartlett's sphericity test were performed to verify the factor ability of the data. Then, EFA was conducted with principal components analysis and varimax rotation to identify the underlying factor structure of the questionnaire. The number of factors extracted was determined by eigenvalue $\geq 1.0$. CFA was performed using the factor structure obtained in EFA. If the cumulative explanatory variation of common factors extracted in the exploratory factor analysis was $>50 \%, \chi^{2} / \mathrm{df}$ in the confirmatory factor analysis was $<3$, the root mean square error of approximation (RMSEA) was $<0.05$, confirmatory fit index (CFI), goodness-of fit index (GFI), incremental fit index (IFI), Tucker-Lewis index (TLI) were $>0.9$, the construct validity of the questionnaire was considered good. ${ }^{32}$

The item-level content validity index (I-CVI), scalelevel content validity index/universal agreement (S-CVI/ UA), and scale-level content validity index/average (S-CVI/AVE), which calculated from expert consultation was utilized to quantify content validity. 10 experts evaluated the relevance of each item and rated using a 4-point Likert scale $(1=$ not relevant, $2=$ somewhat relevant, $3=$ quite relevant, $4=$ highly relevant). According to Polit et $\mathrm{al}^{34}{ }^{34}$ it was recommended that I-CVI $\geq 0.78, \mathrm{~S}-\mathrm{CVI} / \mathrm{UA}$ $\geq 0.8$, and $\mathrm{S}-\mathrm{CVI} / \mathrm{AVE} \geq 0.9$ indicated a good content validity.

Internal consistency reliability, split-half reliability, and test-retest reliability were used to evaluate the questionnaire reliability. Cronbach's $\alpha$ coefficients were estimated to examine internal consistency (criterion 0.70 ). The splithalf reliability of the questionnaire was tested using Guttman split-half coefficient (criterion 0.70). The testretest reliability was assessed by the randomly selected participants completing questionnaires twice within a mean interval of two weeks (criterion 0.70). ${ }^{32}$

\section{Statistical Analysis}

Statistical analysis was performed using the SPSS 25.0 and Amos 23.0. The data was divided into two samples randomly. Sample $1(\mathrm{n}=167)$ was training dataset and sample $2(n=152)$ was confirmatory dataset. Sample 1 was used for item analysis and EFA, while sample 2 was used for CFA, reliability and validity test. The continuous variables were described by mean and standard deviation and the categorical variables were described by frequency and percentage. Statistical significance for all analyses was set at $P<0.05$.

\section{Results}

\section{Characteristics of the Participants}

A total of 326 questionnaires were distributed, and 319 were effectively collected, representing an effective response rate of $97.85 \%$. The 319 participants had a mean age of $33.52 \pm 9.12$ years. Most participants $(63.32 \%)$ were female. Nearly half of participants had sleeve gastrectomy $(49.84 \%)$ or sleeve gastrectomy plus surgery $(42.32 \%)$, with the remainder reporting gastric bypass surgery (7.84\%). Most participants $(65.83 \%)$ had comorbidities and the average BMI was $27.58 \pm 6.65 \mathrm{~kg} /$ $\mathrm{m}^{2}$. All participants underwent bariatric surgery at least 3 months in the past. The basic characteristics of the participants are shown in Table 1.

\section{Item Analysis and Selection}

Items were selected according to six indexes including critical ratio, standard deviation, item-total correlation or corrected item-total correlation coefficient, Cronbach's $\alpha$ after item deletion, factor loading value, and communalities value. The item that met at least three of the above six criteria for deletion was deleted. The overall Cronbach's $\alpha$ for the questionnaire was 0.931 . No items were deleted after the analysis according to the item deletion criteria and all items were included for further analysis. The results of items analysis are presented in Table 2.

\section{Validity Analysis Construct Validity}

According to the KMO and Bartlett's sphericity test, the KMO value was 0.864 and the Bartlett's sphericity test was significant $\left(\chi^{2}=3536.197, P<0.001\right)$, indicating the appropriateness for EFA. After principal components analysis and varimax rotation, four factors with eigenvalues $>1$ were identified, accounting for $59.98 \%$ of the cumulative 
Table I Characteristics of the Participants $(n=319)$

\begin{tabular}{|c|c|c|}
\hline Characteristics & Category & $\begin{array}{c}\text { Means } \pm S D \\
( \pm s) / n(\%)\end{array}$ \\
\hline Age(years) & & $33.52 \pm 9.12$ \\
\hline \multirow[t]{2}{*}{ Gender } & Male & $117(36.68 \%)$ \\
\hline & Female & $202(63.32 \%)$ \\
\hline \multirow[t]{3}{*}{ Education } & Junior school or less & $52(16.30 \%)$ \\
\hline & High school & $75(23.51 \%)$ \\
\hline & College or above & $192(60.19 \%)$ \\
\hline \multirow[t]{3}{*}{ Occupational status } & Employed & $222(69.59 \%)$ \\
\hline & Unemployed & $74(23.20 \%)$ \\
\hline & Retired & $23(7.21 \%)$ \\
\hline \multirow[t]{3}{*}{ Marital status } & Married & $188(58.93 \%)$ \\
\hline & Unmarried & $112(35.11 \%)$ \\
\hline & Divorce or widowhood & $19(5.96 \%)$ \\
\hline \multirow[t]{4}{*}{ Type of surgery } & LSG & $159(49.84 \%)$ \\
\hline & LRYGB & $25(7.84 \%)$ \\
\hline & LSG-JJB & $62(19.44 \%)$ \\
\hline & LSG-DJB & $73(22.88 \%)$ \\
\hline \multirow[t]{2}{*}{ Comorbidity } & Yes & $210(65.83 \%)$ \\
\hline & No & $109(34.17 \%)$ \\
\hline $\mathrm{BMI}\left(\mathrm{kg} / \mathrm{m}^{2}\right)$ & & $27.58 \pm 6.65$ \\
\hline \multirow{4}{*}{$\begin{array}{l}\text { Time since } \\
\text { surgery(months) }\end{array}$} & $3-<6$ & $82(25.71 \%)$ \\
\hline & $6-<12$ & $104(32.60 \%)$ \\
\hline & $12-<24$ & $63(19.75 \%)$ \\
\hline & $\geq 24$ & $70(21.94 \%)$ \\
\hline
\end{tabular}

Abbreviations: LSG, laparoscopic sleeve gastrectomy; LRYGB, laparoscopic Rouxen-Y gastric bypass; LSG-JJB, jejunojejunal bypass with laparoscopic sleeve gastrectomy; LSG-DJB duodenal-jejunal bypass with laparoscopic sleeve gastrectomy.

variance (Table 3). The item "When I am in a good mood, I can adhere to the diet" which had dual loadings (loadings greater than 0.40 on two factors) was deleted. The factors were self-efficacy ( 7 items), social influence (10 items), intention (4 items), and attitude (7 items). Table 4 lists the factors loading of the ASEQBS based on the exploratory factor analysis after deleting the item.

The results of the confirmatory factor analysis $(\mathrm{N}=152)$ found an optimal model that included 28 items spread among the previously identified four factors. The model fit statistics indicated a good fit of the theoretical model to the empirical model: $\chi^{2} / \mathrm{df}=1.517, \mathrm{CFI}=0.936, \mathrm{GFI}=0.928$, $\mathrm{IFI}=0.937, \mathrm{TLI}=0.923$, RMSEA $=0.049$. These results provide support for the structural validity of the four-factor ASEQBS.

\section{Content Validity}

According to experts' evaluation, the I-CVI was between 0.800 and 1.000. The S-CVI/UA and S-CVI/Ave was $0.857,0.979$, respectively.

\section{Reliability Analysis}

Cronbach's $\alpha$ for the 28-item ASEQBS in sample 2 $(\mathrm{N}=152)$ was 0.907 , indicating good internal consistency. Cronbach's $\alpha$ obtained for each dimension was from 0.807 to 0.935 . The split-half coefficient value for all domains was 0.774 and each dimension was from 0.701 to 0.923 , which revealed acceptable internal consistency among items in the questionnaire. In terms of the 2-week testretest reliability assessment, the intra-class correlation coefficient for the total questionnaire was 0.922 and the four domains ranged from 0.844 to 0.923 . These results suggested satisfactory reliability and stability of the 28 item ASEQBS (Table 5).

\section{Discussion}

To the best of our knowledge, the current study is a novel one to use the ASE model as a theoretical framework to develop and validate a questionnaire to assess the determinants of dietary adherence among patients after bariatric surgery. In summary, the final version of the ASEQBS consisted of 28 items, which showed good psychometric properties in a Chinese population. The validity and reliability, including construct validity, content validity, and internal consistency, indicated that the psychometric properties of the ASEQBS were shown to be appropriate. Each item is scored from 1 to 5 to provide the scores of each dimension. A linear transformation was used to calculate scores ranging from 28 to 140, where higher scores in each dimension indicate better intention, attitude, social influence, or self-efficacy.

While bariatric surgery is the most effective and sustainable treatment available for severe obesity, weight regain is still common. It has been reported that approximately $5 \%-20 \%$ of individuals who underwent bariatric surgery fail to maintain sufficient weight loss. ${ }^{35}$ In Wakayama et al's study, ${ }^{36}$ they found that poor dietary adherence at 6 months postoperatively was a significant predictor of higher BMIs. Yanos et $\mathrm{al}^{37}$ also found that dietary adherence was inversely associated with the likelihood of significant weight regain. In addition, poor dietary adherence has also been proven to be linked to a variety of adverse postoperative outcomes, including gastrointestinal symptoms, vitamin D deficiency, anemia, and poor weight outcomes. ${ }^{38-40}$ As dietary adherence is fundamental to medical and surgical outcomes, it is important for patients to adhere to a specific healthy diet for long-term weight loss maintenance. 
Table 2 Item Analysis Results $(n=167)$

\begin{tabular}{|c|c|c|c|c|c|c|c|}
\hline Item & CR & CV & ITC & Cronbach's $\alpha$ After Item Deletion\# & Communalities & Factor Loading & Included Items \\
\hline I & $5.722^{* *}$ & 0.688 & $0.425^{* *}$ & 0.930 & 0.246 & 0.496 & $\sqrt{ }$ \\
\hline 2 & $9.582 * *$ & 0.667 & $0.595 * *$ & 0.928 & 0.474 & 0.689 & $\sqrt{ }$ \\
\hline 3 & $9.873^{* *}$ & 0.691 & $0.575^{* *}$ & 0.928 & 0.456 & 0.676 & $\sqrt{ }$ \\
\hline 4 & $8.844^{* *}$ & 0.678 & $0.586 * *$ & 0.928 & 0.458 & 0.676 & $\sqrt{ }$ \\
\hline 5 & $7.1311^{* *}$ & 0.694 & $0.525 * *$ & 0.929 & 0.313 & 0.560 & $\sqrt{ }$ \\
\hline 6 & $9.439 * *$ & 0.876 & $0.624 * *$ & 0.928 & 0.408 & 0.639 & $\sqrt{ }$ \\
\hline 7 & $6.256^{* *}$ & 0.739 & $0.462^{* *}$ & 0.930 & 0.237 & 0.487 & $\sqrt{ }$ \\
\hline 8 & $3.214^{* *}$ & 0.800 & 0.316 ** & 0.931 & 0.208 & 0.429 & $\sqrt{ }$ \\
\hline 9 & $5.242^{* *}$ & 0.924 & 0.466 ** & 0.930 & 0.247 & 0.497 & $\sqrt{ }$ \\
\hline 10 & $7.080^{* *}$ & 1.016 & $0.505^{* *}$ & 0.929 & 0.308 & 0.555 & $\sqrt{ }$ \\
\hline II & $10.199 * *$ & 0.825 & $0.67 * *$ & 0.927 & 0.439 & 0.662 & $\sqrt{ }$ \\
\hline 12 & $6.53 \mathrm{I} * *$ & 1.008 & $0.547^{* *}$ & 0.929 & 0.267 & 0.517 & $\sqrt{ }$ \\
\hline 13 & $7.093^{* *}$ & 1.027 & $0.586 * *$ & 0.929 & 0.289 & 0.538 & $\sqrt{ }$ \\
\hline 14 & $7.128^{* *}$ & 1.029 & $0.562^{* *}$ & 0.929 & 0.250 & 0.500 & $\sqrt{ }$ \\
\hline 15 & $7.023^{* *}$ & 0.878 & $0.49 I^{* *}$ & 0.930 & 0.200 & 0.447 & $\sqrt{ }$ \\
\hline 16 & $7.160^{* *}$ & 0.763 & $0.584 * *$ & 0.929 & 0.306 & 0.553 & $\sqrt{ }$ \\
\hline 17 & $6.857^{* *}$ & 0.881 & $0.55 I^{* * *}$ & 0.929 & 0.242 & 0.492 & $\sqrt{ }$ \\
\hline 18 & 8.656 ** & 0.723 & $0.60 I^{* *}$ & 0.929 & 0.318 & 0.564 & $\sqrt{ }$ \\
\hline 19 & $7.55 I^{* *}$ & 1.160 & $0.579 * *$ & 0.929 & 0.273 & 0.522 & $\sqrt{ }$ \\
\hline 20 & $8.133^{* *}$ & 1.136 & $0.629 * *$ & 0.928 & 0.349 & 0.591 & $\sqrt{ }$ \\
\hline 21 & $8.982^{* *}$ & 1.234 & $0.629 * *$ & 0.929 & 0.337 & 0.581 & $\sqrt{ }$ \\
\hline 22 & $10.872^{* *}$ & 0.924 & $0.717^{* *}$ & 0.927 & 0.537 & 0.733 & $\sqrt{ }$ \\
\hline 23 & $9.724 * *$ & 0.963 & $0.702^{* *}$ & 0.927 & 0.507 & 0.712 & $\sqrt{ }$ \\
\hline 24 & $9.417^{* *}$ & 0.928 & $0.679 * *$ & 0.927 & 0.493 & 0.702 & $\sqrt{ }$ \\
\hline 25 & $5.876 * *$ & 0.813 & $0.46 I^{* *}$ & 0.930 & 0.209 & 0.458 & $\sqrt{ }$ \\
\hline 26 & $|0.77| * *$ & 0.954 & $0.68 I^{* *}$ & 0.927 & 0.483 & 0.695 & $\sqrt{ }$ \\
\hline 27 & $\left.12.18\right|^{* *}$ & 0.979 & $0.706 * *$ & 0.926 & 0.551 & 0.743 & $\sqrt{ }$ \\
\hline 28 & $12.120 * *$ & 0.919 & $0.725^{* *}$ & 0.926 & 0.567 & 0.753 & $\sqrt{ }$ \\
\hline 29 & $10.058^{* *}$ & 0.779 & $0.690^{* *}$ & 0.927 & 0.508 & 0.712 & $\sqrt{ }$ \\
\hline
\end{tabular}

Notes: ${ }^{*}$ Overall Cronbach's $\alpha$ for the scale was $0.931 ;{ }^{* * P}<0.001$; ${ }^{\vee}$ the item were included for further analysis.

Abbreviations: CR, critical ratio; CV, coefficient of variation; ITC, item-total correlation.

Identifying the possible determinants becomes a critical measure to improve dietary adherence. Previous studies have revealed that psychosocial factors were associated with dietary adherence but they did not use a specific, reliable, and valid measurement. ${ }^{41,42}$ In this study, we developed a 28-item questionnaire with high reliability and good construct validity to measure the determinants of dietary adherence based on the ASE model. A systematic approach has been adopted to ensure the scientific process and robustness of the results. When developing questionnaires, semi-structured interviews and the Delphi method were used to produce the initial items. Besides, participant inclusion in the phase of pilot study might have improved the resulting questionnaire's acceptability and relevance for the target group. Our rigorous development procedure and psychometric evaluation ensured that the ASEQBS is effective and convincing.
Construct validity refers to the degree to which an instrument fully assesses or measures the construct of interest. $^{32}$ In this study, exploratory factor analysis and confirmatory factor analysis were conducted to determine factor structure. Construct validity of the ASEQBS was approved as we found the four-factor solution as the clearest pattern of factor loadings, which accounted for a large proportion $(59.98 \%)$ of all variance between the items. In confirmatory factor analysis, the indexes including $\chi^{2} / \mathrm{df}$, GFI, CFI, IFI, TLI, RMSEA suggested the data fit the model well. The findings supported the conceptual framework of the ASE model which encompasses the four constructs of self-efficacy, social influence, intention, and attitude. Content validity refers to the extent to which the items of an instrument adequately represent the concept to be measured. ${ }^{43}$ By calculating the content validity index, it was found that the I-CVI of each item was $>0.78, \mathrm{~S}-\mathrm{CVI} /$ 
Table 3 Contribution Rates of Characteristic Root Variance and Cumulative Variance of the Factors $(n=167)$

\begin{tabular}{|l|c|c|c|c|}
\hline Factor & Name & Eigenvalue & Vaiance Contribution Rate (\%) & Cumulative Variance Contribution Rate(\%) \\
\hline Factor 1 & Self-efficacy & 10.483 & 36.147 & 36.147 \\
Factor 2 & Social influence & 2.924 & 10.081 & 46.229 \\
Factor 3 & Intention & 2.479 & 8.549 & 54.778 \\
Factor 4 & Attitude & 1.509 & 5.205 & 59.983 \\
\hline
\end{tabular}

Table 4 Factors Loading of the ASEQBS Based on the Exploratory Factor Analysis After Deleting the Item ( $\mathrm{n}=167)$

\begin{tabular}{|c|c|c|c|c|}
\hline Item & $\begin{array}{c}\text { Factor } \\
\text { I }\end{array}$ & $\begin{array}{c}\text { Factor } \\
2\end{array}$ & $\begin{array}{c}\text { Factor } \\
3\end{array}$ & $\begin{array}{c}\text { Factor } \\
4\end{array}$ \\
\hline I. I am willing to adhere to the diet & 0.095 & 0.042 & $0.68 \mathrm{I}$ & 0.326 \\
\hline 2. I plan to adhere to the diet & 0.236 & 0.168 & 0.859 & 0.25 \\
\hline 3. I intend to adhere to the diet & 0.263 & 0.153 & 0.825 & 0.233 \\
\hline 4. I would like to make the effort to adhere to the diet & 0.273 & 0.169 & 0.835 & 0.187 \\
\hline 5. I think adhering to the diet make my gastrointestinal tract comfortable & 0.099 & 0.23 & 0.218 & 0.675 \\
\hline 6. I think adhering to the diet make me feel good & 0.169 & 0.231 & 0.388 & 0.595 \\
\hline 7. I think adhering to the diet is beneficial to my weight loss & 0.254 & 0.047 & 0.062 & 0.654 \\
\hline $\begin{array}{l}\text { 8. I think adhering to the diet is beneficial to my disease remission(eg diabetes, polycystic ovary } \\
\text { syndrome, fatty liver, obstructive sleep apnea syndrome) }\end{array}$ & 0.032 & -0.013 & 0.042 & 0.694 \\
\hline 9. I think the diet tastes delicious & 0.088 & 0.227 & 0.209 & 0.579 \\
\hline 10. I think it is difficult to cook according to the dietary recommendations & 0.275 & 0.156 & 0.221 & 0.526 \\
\hline II. I think adhering to the diet make my life interesting & 0.253 & 0.322 & 0.263 & 0.547 \\
\hline 12. I think the pressure from my family can make me adhere the diet & 0.125 & 0.666 & 0.154 & 0.069 \\
\hline 13. I think the pressure from my friends can make me adhere the diet & 0.13 & 0.697 & 0.079 & 0.128 \\
\hline 14. I think the pressure from my fellow patients can make me adhere the diet & 0.047 & 0.71 & 0.1 & 0.105 \\
\hline 15. I think the pressure from healthcare personnel can make me adhere the diet & 0.07 & 0.431 & 0.158 & 0.225 \\
\hline 16. My family is supportive of me to adhere to the diet & 0.221 & 0.688 & -0.075 & 0.189 \\
\hline 17. My friends are supportive of me to adhere to the diet & 0.165 & 0.795 & -0.203 & 0.119 \\
\hline 18. My fellow patients is supportive of me to adhere to the diet & 0.272 & 0.622 & -0.056 & 0.196 \\
\hline 19. My family can cook food for me according to the dietary recommendations & 0.116 & 0.616 & 0.179 & 0.118 \\
\hline 20. My family had made a healthy diet change & 0.179 & 0.67 & 0.288 & 0.023 \\
\hline 21. My friends had made a healthy diet change & 0.204 & 0.663 & 0.272 & -0.015 \\
\hline 22. My fellow patients adhered to the diet & 0.808 & 0.226 & 0.189 & 0.114 \\
\hline 23. When I am depressed (or down), I can adhere to the diet & 0.826 & 0.228 & 0.124 & 0.106 \\
\hline 24. When I am tired, I can adhere to the diet & 0.882 & 0.103 & 0.151 & 0.123 \\
\hline 25. When I am hungry, I can adhere to the diet & 0.825 & 0.223 & 0.121 & 0.058 \\
\hline 26. When I am in a social situation (such as at a party), I can adhere to the diet & 0.767 & 0.214 & 0.183 & 0.218 \\
\hline 27. When I go out (such as on a business trip or travel), I can adhere to the diet & 0.809 & 0.21 & 0.162 & 0.209 \\
\hline 28. When the season or the weather changes, I can adhere to the diet & 0.715 & 0.146 & 0.137 & 0.325 \\
\hline
\end{tabular}

Note: Bold font represents the factor loading value of the item on its factor.

$\mathrm{UA}$ and S-CVI/Ave were $>0.8$, which indicated that the questionnaire could evaluate the content needed to be measured well. The results of reliability analysis indicated that the total Cronbach's $\alpha$ of ASEQBS was 0.920, suggesting the ASEQBS had good internal consistency. The test-retest reliability refers to the temporal stability of a questionnaire. In this study, the correlation coefficient of ASEQBS was 0.922, indicating that the questionnaire was stable.
The ASE model has been used to understand dietary behaviors in previous studies. However, there are many differences in its application and no model instrument has been validated in the Chinese population, which contributed to no exemplary approaches to follow. In this study, the ASE model has been applied as a theoretical framework to develop the questionnaire, which provided a valid instrument to promote dietary adherence in post-bariatric patients. As proposed by the ASE model, psychosocial 
Table 5 Internal Consistency and Test-Re-Test Reliability Analysis Results $(n=152)$

\begin{tabular}{|l|c|c|c|}
\hline Dimension & Cronbach's $\alpha$ & Spearman-Brown Coefficient & Test-Retest Reliablity \\
\hline Intention & 0.907 & 0.923 & $0.859 * *$ \\
Attitude & 0.807 & 0.701 & $0.844^{* *}$ \\
Social influence & 0.857 & 0.758 & $0.923^{* *}$ \\
Self-efficacy & 0.935 & 0.875 & $0.889 * *$ \\
ASEQBS & 0.920 & 0.774 & $0.922^{* *}$ \\
\hline
\end{tabular}

Notes: $* * p<0.01$.

factors, including intention, attitude, social influence, and self-efficacy are major determinants of health-related behavior. ${ }^{44}$ Furthermore, the behavioral intention may be a mediator between the influence of attitude, social influence, self-efficacy, and behavior. ${ }^{24}$ After developing the theory-supported questionnaire, it is possible to understand the mechanism of specific behavior and identify factors necessary to change health-related behavior. What's more, an understanding of the determinants that cause poor dietary adherence among post-bariatric patients is essential for health professionals to develop interventions. For instance, health professionals could not only fully inform patients of the benefits of following dietary recommendations to enhance their positive attitude, but also pay attention to patients with negative attitudes to help them identify the barriers to improving dietary adherence.

\section{Implication}

This study developed a new theory-based tool to measure the determinants of dietary adherence in Chinese postbariatric patients. It is useful to assess intention, attitude, social influence, and self-efficacy, which provides an appropriate instrument for future research. The relationship between the ASE model constructs and dietary adherence needs to be explored and understood by using this questionnaire. Besides, this questionnaire would assist in identifying relevant determinants and in planning theorybased interventions that might result in improving dietary adherence in health behavior change programs. This study also provides guidance on how to develop effective measurements based on the ASE model for future work.

In the clinical setting, nutritionists and nurses play important roles to provide dietary recommendations and intervention in hospitals and other healthcare centers. This questionnaire can help to understand patients' intention, attitude, social influence, and self-efficacy, which provide the basis to change their irrational eating behavior and improve dietary adherence during the postoperative follow-up. Nutritionists and nurses can intervene early as part of a multidisciplinary healthcare team to assess dietary adherence and related factors and to provide tailored health education with the goal of improving surgical outcomes.

\section{Limitation}

Some limitations need to be acknowledged. Firstly, the participants in this study were all recruited from one hospital in Jiangsu Province with convenience sampling, which may limit the representativeness of the findings. In the future, samples from different regions, countries, and settings need to be selected to test the psychometric properties of the questionnaire. Furthermore, in conducting our interviews and analysis based on the ASE model, constructs may have limited the emergence and identification of determinants not contemplated in this theory. Hence, it is suggested that future studies could focus on more determinants to further improve dietary adherence. Finally, for the data analysis, the criterion validity was not tested because there was no appropriate criterion to measure the determinants of dietary adherence for Chinese postbariatric patients. Therefore, future research to evaluate the criterion validity of the questionnaire should be explored and a further validation study could promote use of the questionnaire.

\section{Conclusion}

The final 28-item questionnaire provides a theory-based instrument for evaluating the determinants of dietary adherence among patients after bariatric surgery, which indicated appropriate validity and reliability. The ASEQBS contained 4 dimensions consistent with the ASE model, which were intention, attitude, social influence, and self-efficacy. Given the importance of following dietary recommendations in improving weight loss outcomes, the development of this questionnaire demonstrates an effective step toward evaluating the facilitators and 
barriers of dietary adherence. What's more, it is useful to apply a reliable and valid tool to understand the behavior of interest correctly, as well as determine the relative importance and relationship between constructs. It also provides a means for researchers and clinical staff to design and evaluate the efficacy of tailored dietary intervention programs.

\section{Funding}

1. Project of "Nursing Science" Funded by the Priority Academic Program Development of Jiangsu Higher Education Institutions (2018, No.87). 2. Project"The exploration of trajectories and intervention program of frailty for gastric cancer survivors based on the health ecology theory" supported by National Natural Science Foundation of China (NSFC) (No: 82073407). 3. Project "Early warning and intervention of childhood and adolescent obesity in the context of big data: Development and demonstrative application of iNATURE intelligent platform based on hospital, community, and family linkage" supported by the Social Development Fund of Jiangsu Province (No: BE2021722).

\section{Disclosure}

The authors declare that they have no competing interests.

\section{References}

1. World Health Organization. Obesity and overweight. Available from: https://www.who.int/en/news-room/fact-sheets/detail/obesity-andoverweight. Accessed October 9, 2021.

2. Piché ME, Tchernof A, Després JP, Phenotypes O. Diabetes, and cardiovascular diseases. Circ Res. 2020;126(11):1477-1500. doi:10.1161/CIRCRESAHA.120.316101

3. Avgerinos KI, Spyrou N, Mantzoros CS, Dalamaga M. Obesity and cancer risk: emerging biological mechanisms and perspectives. Metabolism. 2019;92:121-135. doi:10.1016/j.metabol.2018.11.001

4. Blüher M. Obesity: global epidemiology and pathogenesis. Nat Rev Endocrinol. 2019;15(5):288-298. doi:10.1038/s41574-019-0176-8

5. The State Council Information Office of the People's Republic of China. Report on nutrition and chronic diseases of Chinese residents. Available from: http://www.scio.gov.cn/xwfbh/xwbfbh/wqfbh/42311/ 44583/wz44585/Document/1695276/1695276.htm. Accessed October 9, 2021.

6. NCD Risk Factor Collaboration (NCD-RisC). Trends in adult body-mass index in 200 countries from 1975 to 2014: a pooled analysis of 1698 population-based measurement studies with 19.2 million participants. Lancet. 2016;387(10026):1377-1396. doi:10.1016/ S0140-6736(16)30054-X

7. Guraya SY, Strate T. Surgical outcome of laparoscopic sleeve gastrectomy and Roux-en-Y gastric bypass for resolution of type 2 diabetes mellitus: a systematic review and meta-analysis. World J Gastroenterol. 2020;26(8):865-876. doi:10.3748/wjg.v26.i8.865

8. Angrisani L, Santonicola A, Iovino P, et al. IFSO worldwide survey 2016: primary, endoluminal, and revisional procedures. Obes Surg. 2018;28(12):3783-3794. doi:10.1007/s11695-018-3450-2
9. Kessler Y, Adelson D, Mardy-Tilbor L, et al. Nutritional status following One Anastomosis Gastric Bypass. Clin Nutr. 2020;39 (2):599-605. doi:10.1016/j.clnu.2019.03.008

10. Nutrition and Metabolism Collaboration of Chinese Society for Parenteral and Enteral Nutrition, Bariatric Multidisciplinary Team of Peking Union Medical College Hospital. The consensus on nutritional and multi-disciplinary management for bariatric surgery. Chin J Surg. 2018;56(2):81-90. doi:10.3760/cma.j.issn.0529-5815.2018.02.001

11. Mechanick JI, Apovian C, Brethauer S, et al. Clinical Practice Guidelines for the Perioperative Nutrition, Metabolic, and Nonsurgical Support of Patients Undergoing Bariatric Procedures - 2019 Update: cosponsored by American Association of Clinical Endocrinologists/ American College of Endocrinology, The Obesity Society, American Society for Metabolic and Bariatric Surgery, Obesity Medicine Association, and American Society of Anesthesiologists. Obesity. 2020;28(4):O1-o58. doi:10.1002/oby.22719

12. Tabesh MR, Maleklou F, Ejtehadi F, Nutrition AZ, Activity P. and prescription of supplements in pre- and post-bariatric surgery patients: a practical guideline. Obes Surg. 2019;29(10):3385-3400. doi:10.1007/s11695-019-04112-y

13. Hood MM, Kelly MC, Feig EH, Webb V, Bradley LE, Corsica J. Measurement of adherence in bariatric surgery: a systematic review. Surg Obes Relat Dis. 2018;14(8):1192-1201. doi:10.1016/j. soard.2018.04.013

14. Sherf Dagan S, Keidar A, Raziel A, et al. Do bariatric patients follow dietary and lifestyle recommendations during the first postoperative year? Obes Surg. 2017;27(9):2258-2271. doi:10.1007/s11695-0172633-6

15. Pontiroli AE, Fossati A, Vedani P, et al. Post-surgery adherence to scheduled visits and compliance, more than personality disorders, predict outcome of bariatric restrictive surgery in morbidly obese patients. Obes Surg. 2007;17(11):1492-1497. doi:10.1007/s11695008-9428-8

16. Li M, Liu Y, Zhang S, et al. Greater China metabolic and bariatric surgery database registry report(2020). Chin J Prac Surg. 2021;5 (41):533-542. doi:10.19538/j.cjps.issn1005-2208.2021.05.08

17. Sutton SM, Magwood GS, Jenkins CH, Nemeth LS, Scoping A. Review of behavioral weight management interventions in overweight/obese African American females. West J Nurs Res. 2016;38 (8):1035-1066. doi:10.1177/0193945916635160

18. De Vries H, Dijkstra M, Kuhlman P. Self-efficacy: the third factor besides attitude and subjective norm as a predictor of behavioural intentions. Health Educ Res. 1988;3(3):273-282. doi:10.1093/her/ 3.3.273

19. De Vries H, Mudde AN, Dijkstra A, Willemsen MC. Differential beliefs, perceived social influences, and self-efficacy expectations among smokers in various motivational phases. Prev Med. 1998;27 (5 Pt 1):681-689. doi:10.1006/pmed.1998.0344

20. De Vries H, Mudde AN. Predicting stage transitions for smoking cessation applying the attitude-social influence-efficacy model. Psychol Health. 2007;13(2):369-385. doi:10.1080/0887044980 8406757

21. Liu X, Cai CF, Yu LP. Research progress on diet adherence of type 2 diabetes patients. Chin J Health Educ. 2019;6(35):538-541. doi:10.16168/j.cnki.issn.1002-9982.2019.06.013

22. Lechner L, De Vries H. Starting participation in an employee fitness program: attitudes, social influence, and self-efficacy. Prev Med. 1995;24(6):627-633. doi:10.1006/pmed.1995.1098

23. Brug J, Debie S, Assema PV, Weijts W. Psychosocial determinants of fruit and vegetable consumption among adults: results of focus group interviews. Appetite. 1995;25(3):285-296. doi:10.1016/j. appet.2013.02.005

24. Pajor EM, Eggers SM, Curfs KCJ, Oenema A, Vries HD. Why do Dutch people use dietary supplements? Exploring the role of socio-cognitive and psychosocial determinants. Appetite. 2017;114:161-168. doi:10.1016/j.appet.2017.03.036 
25. Zhang S, Yan J, Sun Y, Meng F, Chen X. The stages and influencing factors of dietary behaviors in young and middle-aged patients with type 2 diabetes. Chin J Nurs. 2018;53(7):816-821. doi:10.3761/j. issn.0254-1769.2018.07.010

26. Bolman C, Arwert TG, Vollink T. Adherence to prophylactic asthma medication: habit strength and cognitions. Heart Lung. 2011;40 (1):63-75. doi:10.1016/j.hrtlng.2010.02.003

27. Bergh I, Lundin Kvalem I, Risstad H, Sniehotta FF. Preoperative predictors of adherence to dietary and physical activity recommendations and weight loss one year after surgery. Surg Obes Relat Dis. 2016;12(4):910-918. doi:10.1016/j.soard.2015.11.009

28. Ames GE, Heckman MG, Diehl NN, Grothe KB, Clark MM. Further statistical and clinical validity for the Weight Efficacy Lifestyle Questionnaire-Short Form. Eat Behav. 2015;18:115-119. doi:10.1016/j.eatbeh.2015.05.003

29. Elo S, Kyngas H. The qualitative content analysis process. $J A d v$ Nurs. 2008;62(1):107-115. doi:10.1111/j.1365-2648.2007.04569.x

30. Zhu H, Yang N, Ren Z, Zhao K, Jiang X, Xu Q. Influencing factors of dietary adherence among patients following bariatric surgery: a qualitative study. Chin J Nurs. 2021;1(56):56-61.

31. Ni P, Chen J, Liu N. The sample size estimation in quantitative nursing research. Chin J Nurs. 2010;4(45):378-380. doi:10.3761/j. issn.0254-1769.2010.04.037

32. Wu ML. Questionnaire Statistical Analysis Practice: SPSS Operation and Application. Chongqing: Chongqing university press; 2010.

33. Gu F, Lin Z, Shang X, Bian Q, Zhang L, Zhang H. Construction and preliminary application of Symptom Cluster Scale for inflammatory bowel disease. Chin J Nurs. 2020;12(55):1819-1824. doi:10.3761/j. issn.0254-1769.2020.12.011

34. Polit DF, Beck CT, Owen SV. Is the CVI an acceptable indicator of content validity? Appraisal and recommendations. Res Nurs Health. 2007;30(4):459-467. doi:10.1002/nur.20199

35. Mauro M, Papelbaum M, Brasil MAA, et al. Is weight regain after bariatric surgery associated with psychiatric comorbidity? A systematic review and meta-analysis. Obes Rev. 2019;20 (10):1413-1425. doi:10.1111/obr.12907
36. Wakayama L, Nameth K, Adler S, Safer DL. Replication and extension of dietary adherence as a predictor of suboptimal weight-loss outcomes in postbariatric patients. Surg Obes Relat Dis. 2019;15 (1):91-96. doi:10.1016/j.soard.2018.10.029

37. Yanos BR, Saules KK, Schuh LM, Sogg S. Predictors of Lowest Weight and Long-Term Weight Regain Among Roux-en-Y Gastric Bypass Patients. Obes Surg. 2015;25(8):1364-1370. doi:10.1007/ s11695-014-1536-z

38. Sarwer DB, Dilks RJ, West-Smith L. Dietary intake and eating behavior after bariatric surgery: threats to weight loss maintenance and strategies for success. Surg Obes Relat Dis. 2011;7(5):644-651. doi:10.1016/j.soard.2011.06.016

39. Henfridsson P, Laurenius A, Wallengren O, et al. Micronutrient intake and biochemistry in adolescents adherent or nonadherent to supplements 5 years after Roux-en-Y gastric bypass surgery. Surg Obes Relat Dis. 2019;15(9):1494-1502. doi:10.1016/j.soard.2019. 06.012

40. Lim HS, Kim YJ, Lee J, Yoon SJ, Lee B. Establishment of Adequate Nutrient Intake Criteria to Achieve Target Weight Loss in Patients Undergoing Bariatric Surgery. Nutrients. 2020;12(6):1774. doi:10.3390/nu12061774

41. Modi AC, Zeller MH, Xanthakos SA, Jenkins TM, Inge TH. Adherence to vitamin supplementation following adolescent bariatric surgery. Obesity. 2013;21(3):E190-195. doi:10.1002/oby.20031

42. Mahawar KK, Clare K, O'Kane M, Graham Y, Callejas-Diaz L, Carr WRJ. Patient Perspectives on Adherence with Micronutrient Supplementation After Bariatric Surgery. Obes Surg. 2019;29 (5):1551-1556. doi:10.1007/s11695-019-03711-Z

43. Shi J, Mo X, Sun Z. Content validity index in scale development. $J$ Cent South Univ. 2012;37(2):49-52. doi:10.3969/j.issn.16727347.2012.02.007

44. Pandey S, Budhathoki M, Yadav DK. Psychosocial Determinants of Vegetable Intake Among Nepalese Young Adults: an Exploratory Survey. Front Nutr. 2021;8:688059. doi:10.3389/fnut.2021.688059
Patient Preference and Adherence

\section{Publish your work in this journal}

Patient Preference and Adherence is an international, peer-reviewed, open access journal that focusing on the growing importance of patient preference and adherence throughout the therapeutic continuum. Patient satisfaction, acceptability, quality of life, compliance, persistence and their role in developing new therapeutic modalities and compounds to optimize clinical outcomes for existing disease states are major areas of interest for the journal. This journal has been accepted for indexing on PubMed Central. The manuscript management system is completely online and includes a very quick and fair peer-review system, which is all easy to use. Visit http:/ www.dovepress.com/testimonials.php to read real quotes from published authors. 